\title{
A measure to predict critical errors in open emergency surgery
}

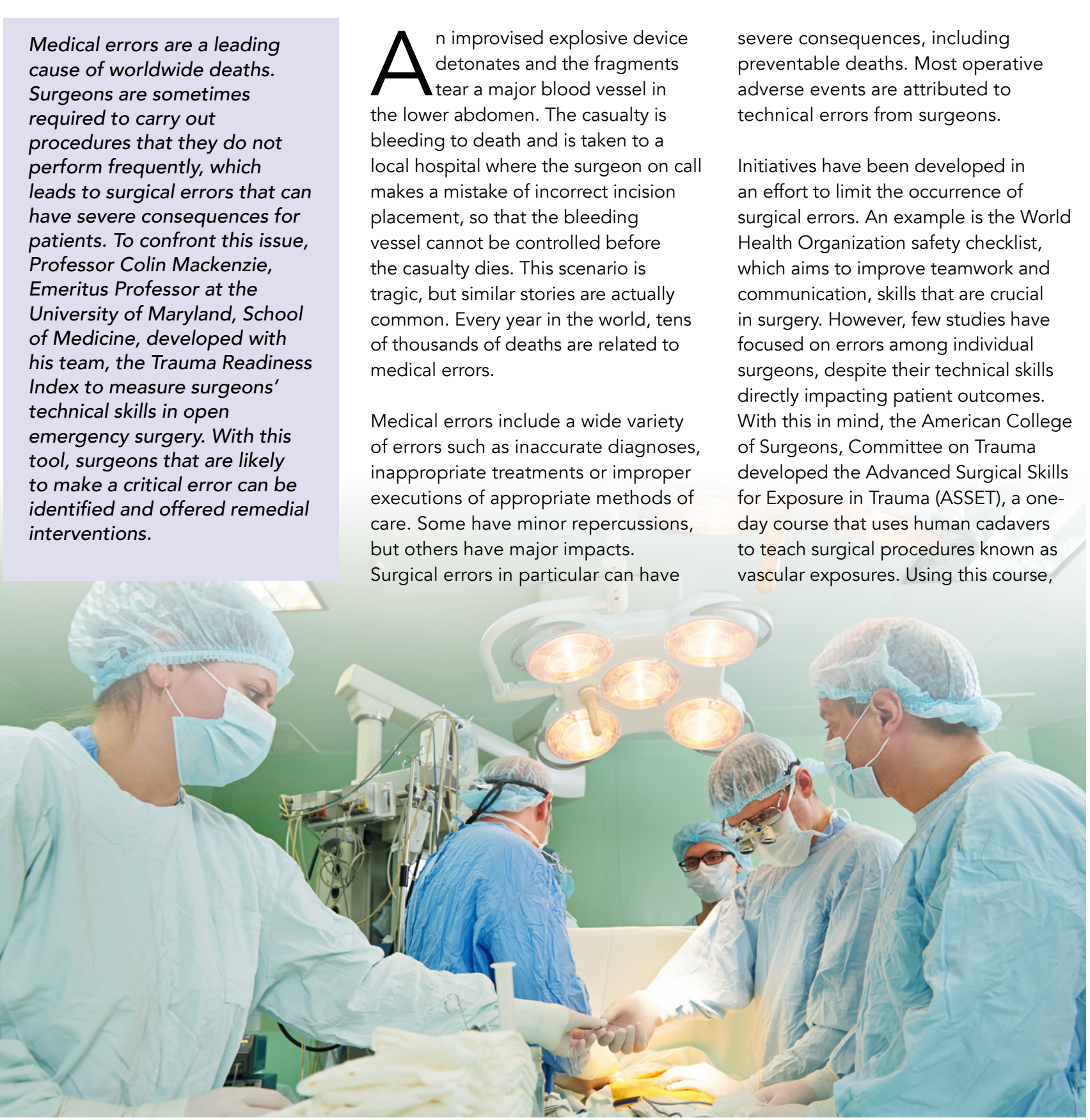

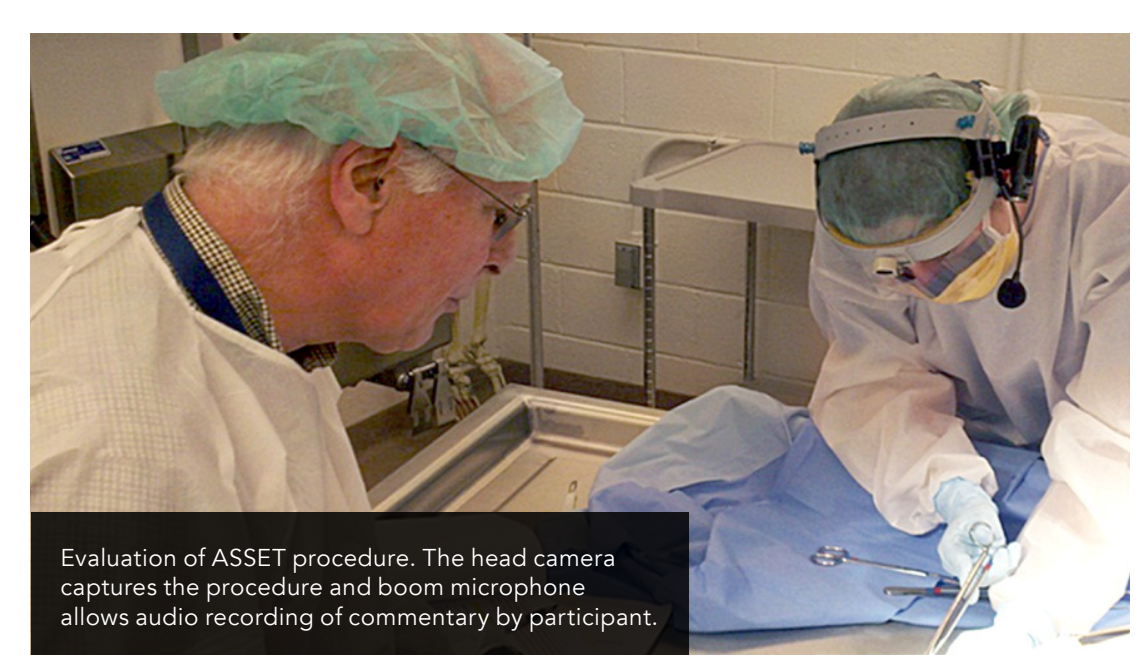

Worldwide, every year, tens of thousands of deaths are related to medical errors.

Professor Mackenzie and his team developed the Trauma Readiness Index

\section{THE ASSET COURSE}

surgeons are no exception to the rule. The issue is that their errors have more severe and immediate consequences than those of the average physician. A surgical error does not necessarily mean a surgeon is incompetent. Surgical competency tis such as good decision-making, efficient teamwork and communication (with surgical, anaesthetic, nursing, and other essential staff members), and technical skills. More often than not, errors of various gravity occur during the process,
even for the simplest of cases.

One of the reasons why surgical errors occur is the infrequent basis on which specific surgical procedures ar performed: when a trauma patient arrives for emergency surgery, surgeons do not have time to go back to their textbooks to refresh their memories; repeated training and courses like ASSET can be beneficial for these infrequently performed emergency procedures.

The ASSET course is open to any surgeon wanting to polish up their anatonical knowledge and technical skills. It uses cadaver surgical simulat to teach exposure of anatomical structures that, when injured, may life. The course allows surgeons to perform emergency surgical procedures to manage vascular, cardiac and major with major trauma.

To assess the effects of the ASSET course, Professor Mackenzie and his team evaluated the performance of participants before and after the course.

\section{RESIDENTS, PRACTICING}

SURGEONS AND EXPERTS

Three different cohorts of surgeons took part in the study. The main cohort was group of 40 general surgery residents, that is to say qualfed physirins, on their

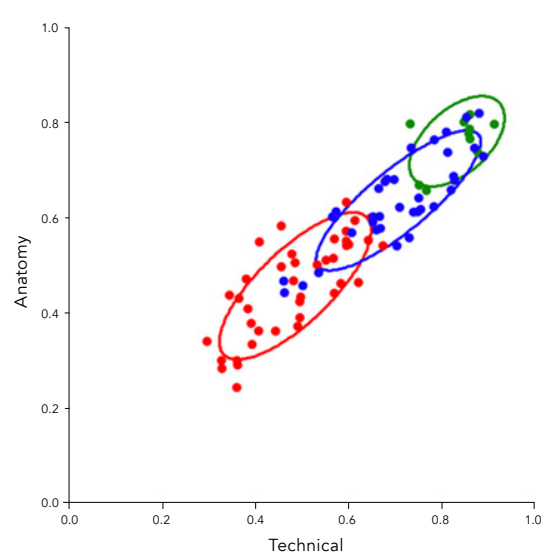

Fig 1: Cluster analysis of correct anatomy
skills and technnical procedural steps for procedures by 40 trainee surgeon befor 10 expert practicing trauma-centre surgeon (green). Cohort centroid is shown by colour
circles The tertiles of performance are sho for each surgeon cohort as within the coloured
circles, above (better performing) the circles circles, above (better performing) the circles
and below the circles (lower performing). average 30 months after ASSET (blue) and

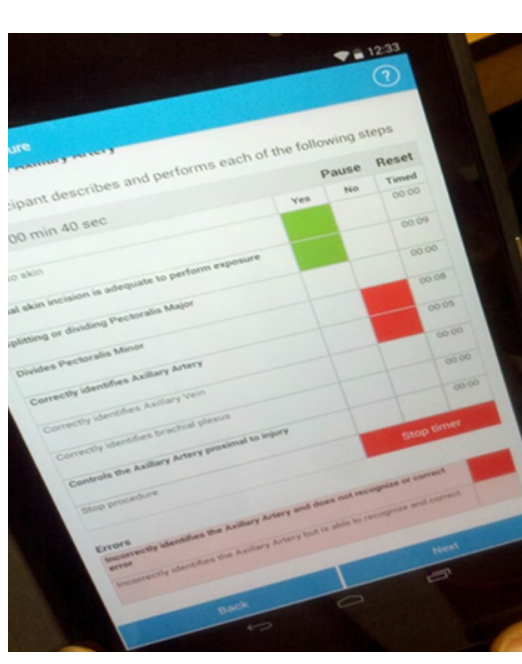

Mobile tablet showing app with
history and evaluation metrics.

way to becoming surgeons. Residents were evaluated a first time before they weceived ASSET traning. They were after attending the course. Finally, to assess skill retention, they were evaluated a third time about 14 months after ASSET training.

The performance of residents was compared to the performance of 34 practicing surgeons who had received ASSET training between 2 to 4 years prior, and of 10 experts who were surgeons and traumatologists from evel 1 trauma centres, hospitals that provide the highest level of surgical care to patients suffering from majo trauma injuries.

FOUR SURGICAL PROCEDURES The technical performance of residents, practicing surgeons and experts procedures. Three of them, called vascular exposures, consisted of exposing (uncovering) three differen arteries of the arms and of the legs, and in placing a ligature around the vessels before the injury to stop the blood flow. The fourth surgical procedure, called fasciotomy, is a procedure that is needed in case of traumas such as bone fractures or crush injuries which cause compression of nerves and blood vessels: the pressure around blood vessels needs to be relieved to restore blood flow and prevent permanent muscle or nerve damage.

Some surgeons in each cohort, including experts, had performed 


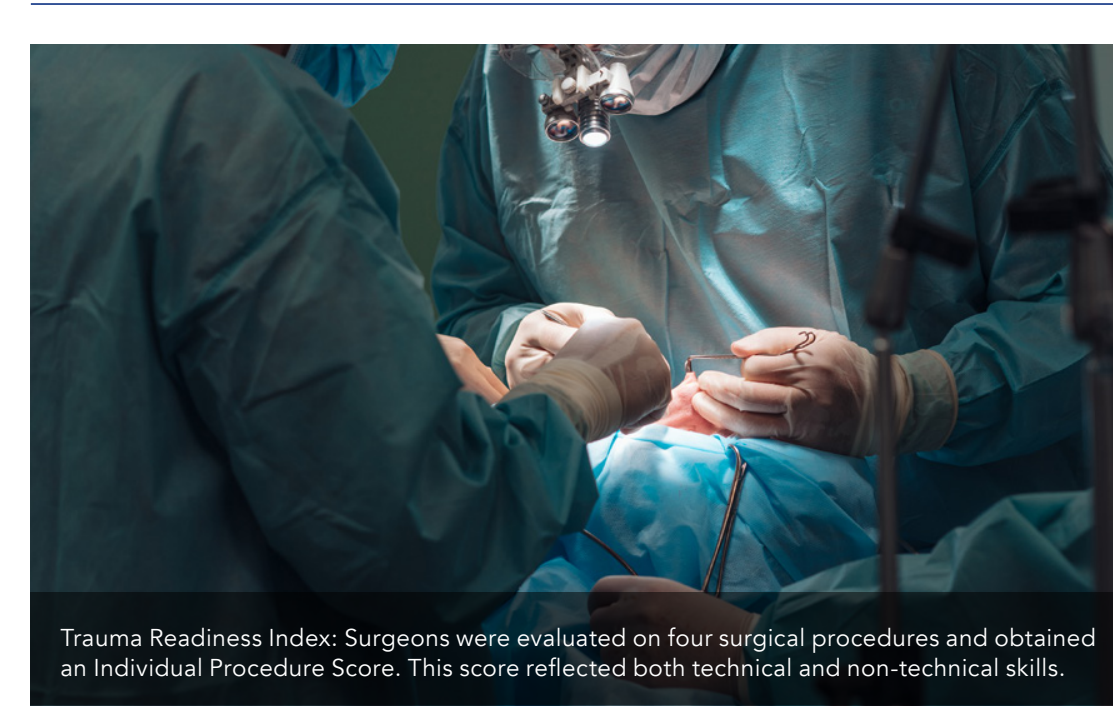

Conducting simple screening tests every two years could limit the number of future critical errors and save lives.

none of these procedures since

ASSET training.

Time to complete each of the procedu was limited. The number of critical technical errors was calculated. Indeed. errors were made as surgeons from all cohorts, including experts, incorrectly placed skin incisions, failed to identity pressure around blood vessels.

\section{TRAUMA READINESS INDEX}

Surgeons were evaluated and for each of the four surgical procedure obtained an Individual Procedure Score. This score, developed using surgeons, reflected both technicat and non-technical skills: besides performing the procedures, surgeons were asked questions about diagnosis, patient management, anatomy and procedural steps that also earned points. Technical skills took into account a proper use of instruments, a correct handling of tissues and an overall success of the procedure. Critical errors that were potentially life-threatening were recorded for each procedure. In addition to the number of errors, error recovery was also assessed: surgeons were sometimes able to recognise and

Trauma Readiness Index (TRI) was a global score corresponding to the sum of after training, TRI was still as high Experts had the highest TRI while experts and residents after training.

The ASSET course also reduces the number of critical errors: before training, the average number of critica number went down to 3.0 within a month after training and further down to 1.6 when they were evaluated 14 months later. In comparison, erro occurrence was 2.8 in practicing surgeons and 1.4 in experts, which means that, 14 months after training, morignificantly before ASSET traiperts. In addity residents, ne procedures without making a sing procedures without making a single (27.5\% of the group) within a month after training and $8(21 \%)$ of them 14 months later made no critical technical error, something that only $20 \%$ of experts achieved.

Finally, the ASSET course also improves the ability to recognise and correct errors. Error recovery is something experts excelled at as they recovered errors 7 times better than residents. before training and practicing surgeons and, even though residents had

improved at recognising and correcting erors aftertrang, experts stllhad about 5 times

TRI, A USEFUL ERROR PREDICTOR One notable finding was that TRI can predict critical errors: residents with ower TRI before ASSET training were those who made critical errors within Low TRI was associated with critical errors also in practicing surgeons and experts: a single evaluation using TRI can predict that an individual surgeon will make a critical error that could be limb or life-threatening

Fig 2: Cluster analysis of correct anatomy
skills and technical procedural steps by san trainee surgeons after ASSET training red; comperison to the same practicing surgeons
(blue) and expert surgeons (green) shown in

Figure 1. Cohort centroid is shown by coloured This finding indicates that TRI can be
circles. Note how the red dots of trainee skills now overlap those of practicing surgeons. The upper tertile of trainee surgeons (red
dots within green circle) are now performing no differently than experts, the majority of
trainees are within the red circle, while 5 of the trainees are within the red circle, whilie 5 of
low performing trainees are shown as the red dots lower than the red circle of their peers
dots low suggesting that these trainees showed no
benefit from the ASSET course training. in need of remedial interventions. Professor Mackenzie and his team suggest that conducting simple screening tests every two years could therefore limit the number of fut

\section{Behind the Research}

Dr Colin
Mackenzie

Research Objectives

Colin Mackenzie's main areas of clinical research include surgeon performance and trauma resuscitation.

\section{Detail}

Colin F. Mackenzie, MD
Suite LL-01, 10 S. Paca Street

Baltimore, MD 21201

Stacy Shackelford MD, FACS, MC 3698 Chambers
Houston, TX 78123

Col (ret) Mark W. Bowyer, MD, FACS Dept of Surgery, 4301 Jones Bridge Rd

Bio

Colin Mackenzie was a Trauma Clinician USA, for over 30 years an the National Study Center for Trauma.

He is now an Emeritus Professor at
the University of Maryland, School of
Medicine.
Funding
The Research and Development
project, conducted by the University of
Maryland, School of Medicine, was made
possible by a cooperative agreement
W81XWH-13-2-0028, awarded and
administered by the U.S. Army Medical
Research \& Materiel Command and
the Congressionally Directed Medical
Research Programs Office at Fort
Detrick, MD (Principal Investigator:
Dr Mackenzie).

Collaborators Col Stacy A. Shackelford, MD, FACS, USAF, MC. Chief, Joint Trauma System, Support Mark W Bowyer, MD, FACS, DMCC, FRCS (Glasg) is the Ben Eiseman Professor of Surgery and Surgical
Director of Simulation at the Uniformed Services University, 'America's Medical School'. Mark is Chief of Trauma and
Combat Surgery at the Uniformed Combat Surgery at the Uniformed Sciences (the military medical school), in trauma education, he is the principle architect of the ASSET course.

\section{References}

Mackenzie C.F., Shackelford S.A., Tisherman S.A., Yang S Puche A., Elster E., Bowyer M.A., and RASP Investigators. Proct org/10.1016/isurg.2019.05.031

Mackenzie C.F., Garofalo E., Puche A., Chen H., Pugh K., Shackelford S., Tisherman S., Henry S., Bowyer M.W. and and fasciotomy among urgical residents beforular expos taining compared with experts. JAMA Surgery, 152(6):58 588. https:///doi.org/10.1016/1.jamcollsurg. 2018.04.028 Mackenzie C.F., Bowyer M.W., Henry S., Isherman S.A.', SA. and RASP Investigators. (2018), Cadaver-Bsed Trauma Procedural Skills Training: Skills Retention 30 Months after Training among Practicing Surgeons in Comparison to Experts
and More Recently Trained Residents. Journal of the American and More Recently Trained Residents. Journal of the America
College of Surgeons, 227:270-279. https://doi.org/10.1016/j. jamcollsurg.2018.04.028

- Mackenzie C.F., Tisherman S., Shackelford S., Sevdalis N. Elster E., Bowyer M.W. (2019). Trauma surgery technical Surgical Education, 76:832-843, https://doico. org /10.1016/

- jurr.2018.10.004 Scoping evidence review on training and skills assessment for

\section{Personal Response}

What sort of remedial interventions would you offer to surgeons who need it? Would you offer them to attend

II The Individual Procedure Scores developed for each of the procedures are based on satisfactory performance steps of a given procedure. Using this assessment tool not only can we identify a surgeon who is not performing at the level of an expert but can also provide individualised remediation plan can be teveloped, tailored to the discrete steps of the procedure and range anyw the anatomy to repeating the individual procedure in a mentored fashion on
repeating the course.

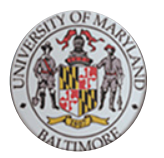

III UNIVERSITY of MARYLAND 